University of Nebraska - Lincoln

DigitalCommons@University of Nebraska - Lincoln

$6-2010$

\title{
Effects of Gd Doping and Oxygen Vacancies on the Properties of EuO Films Prepared via Pulsed Laser Deposition
}

\author{
Xianjie Wang \\ University of Wyoming \\ Pan Liu \\ University of Wyoming \\ Kyle A. Fox \\ University of Wyoming \\ Jinke Tang \\ University of Wyoming, jtang2@uwyo.edu \\ Juan A. Colón Santana \\ University of Nebraska-Lincoln \\ See next page for additional authors
}

Follow this and additional works at: https://digitalcommons.unl.edu/physicsbelashchenko

Wang, Xianjie; Liu, Pan; Fox, Kyle A.; Tang, Jinke; Colón Santana, Juan A.; Belashchenko, Kirill D.; Dowben, Peter A.; and Sui, Yu, "Effects of Gd Doping and Oxygen Vacancies on the Properties of EuO Films Prepared via Pulsed Laser Deposition" (2010). Kirill Belashchenko Publications. 1.

https://digitalcommons.unl.edu/physicsbelashchenko/1

This Article is brought to you for free and open access by the Research Papers in Physics and Astronomy at DigitalCommons@University of Nebraska - Lincoln. It has been accepted for inclusion in Kirill Belashchenko Publications by an authorized administrator of DigitalCommons@University of Nebraska - Lincoln. 


\section{Authors}

Xianjie Wang, Pan Liu, Kyle A. Fox, Jinke Tang, Juan A. Colón Santana, Kirill D. Belashchenko, Peter A. Dowben, and Yu Sui 


\title{
Effects of Gd Doping and Oxygen Vacancies on the Properties of EuO Films Prepared via Pulsed Laser Deposition
}

\author{
Xianjie Wang ${ }^{1}$, Pan Liu ${ }^{1}$, Kyle A. Fox ${ }^{1}$, Jinke Tang ${ }^{1}$, Juan A. Colón Santana ${ }^{2}$, Kirill Belashchenko ${ }^{2}$, \\ Peter A. Dowben ${ }^{2}$, and Yu Sui ${ }^{3}$ \\ ${ }^{1}$ Department of Physics \& Astronomy, University of Wyoming, Laramie, WY 82071 USA \\ ${ }^{2}$ Department of Physics and Astronomy and the Nebraska Center for Materials and Nanoscience, University of Nebraska-Lincoln, \\ Lincoln, NE 68588-0111 USA \\ ${ }^{3}$ Center for Condensed Matter Science and Technology, Department of Physics, Harbin Institute of Technology, \\ Harbin 150001, China
}

\begin{abstract}
We have successfully prepared EuO films on $\mathrm{Si}(100)$ wafers via pulsed laser deposition (PLD). It is well recognized that EuO grows with texture growth along (100) but the addition of $4 \%$ Gd changes the lattice constant and the texture growth to (111) as well as having a profound influence on the magnetic properties. The differences in the effects between Gd doping and oxygen vacancies, both expected to be n-type (donor state) dopants in EuO, are discussed.
\end{abstract}

Index Terms-Doping, EuO, Gd, magnetic semiconductors, oxygen vacancies, pulsed laser deposition.

\section{INTRODUCTION}

$\mathbf{E}$ UROPIUM oxide is a well known ferromagnetic semiconductor, and is a good candidate for spin filter barrier materials [1], [2]. Stoichiometric EuO has a Curie temperature $\left(\mathrm{T}_{\mathrm{C}}\right)$ of $69 \mathrm{~K}$. There is a strong enhancement of the ferromagnetic $\mathrm{T}_{\mathrm{C}}$ by electron doping via rare-earth atoms [3]-[6] or oxygen vacancies [4], [7], [8]. Furthermore, the conductivity of EuO can be tuned to match that of silicon by doping $\mathrm{EuO}$ with oxygen vacancies or with rare-earth elements [3], [9], [10].

Epitaxial or very strongly textured $\mathrm{EuO}(100)$ films can be grown on $\mathrm{Si}$ (100) wafer with high quality interface [11]. The known complication is that the presence of a high oxygen partial pressure at the initial stages of the $\mathrm{EuO}$ film growth leads to formation of $\mathrm{Eu}^{3+}$ (indicative of $\mathrm{Eu}_{2} \mathrm{O}_{3}$ ) at the $\mathrm{Si} / \mathrm{EuO}$ interface [3], [10]. Generally, the investigation and understanding of the properties of europium oxide films may have been hindered by the difficulty in preparing controllable and stable films [4]. Methods for preparing EuO films reported so far include reactive thermal evaporation of Eu and molecular beam epitaxy (MBE) under ultra-high vacuum condition in the presence of oxygen gas [3], [10], [12]-[14].

In this work, we have successfully prepared the high quality europium oxide films on $\mathrm{Si}(100)$ wafer via pulsed laser deposition (PLD) and investigated the magnetic properties of the resulting films. There are changes in the texture growth and lattice constant seen with just a small number of either oxygen vacancies or the inclusion of a small amount of Gd in the PLD grown EuO films. The combination of oxygen vacancies and $\mathrm{Gd}$ doping leads to a dramatic change not only in the $\mathrm{T}_{\mathrm{c}}$ of $\mathrm{EuO}$,

Manuscript received October 30, 2009; revised January 17, 2010; accepted March 12, 2010. Current version published May 19, 2010. Corresponding author: J. Tang (e-mail: jtang2@uwyo.edu).

Color versions of one or more of the figures in this paper are available online at http://ieeexplore.ieee.org.

Digital Object Identifier 10.1109/TMAG.2010.2046314 but unusual heterostructure device characteristics with $\mathrm{Si}(100)$ that are sensitive to an in-plane applied field at room temperature, well above the EuO Curie temperature $\left(\mathrm{T}_{\mathrm{c}}\right)$.

\section{SAMPLE PREPARATION}

The Si wafers were cleaned with diluted HF acid and acetone, and then immediately placed in the vacuum chamber. Before the deposition, the $\mathrm{Si}$ wafers were annealed at $750^{\circ} \mathrm{C}$ in vacuum of $10^{-5}$ Torr under pure- $\mathrm{H}_{2}$ gas in order to further remove the native $\mathrm{SiO}_{2}$ surface layer from the wafers. The annealing times of 30 and 50 min resulted in stoichiometric and oxygen deficient films, respectively. The $\mathrm{EuO}$ and $\mathrm{Gd}$ doped $\mathrm{EuO}$ films were grown using pulsed laser deposition (PLD) in a vacuum of $10^{-5}$ Torr with $\mathrm{H}_{2}$ on these prepared $\mathrm{Si}(100)$ wafers at room temperature. The PLD targets were either Eu (99.9\%) metal or a mixture of $\mathrm{Eu}(99.9 \%)$ and $\mathrm{Gd}(99.9 \%)$ metals, and the purity of $\mathrm{H}_{2}$ gas used during the deposition is $99.995 \%$. To prevent the degradation of $\mathrm{EuO}_{1-\mathrm{x}}$ films when exposed to air, the film were protected in situ by a Pt capping layer, except those films used for device fabrication.

X-ray diffraction (XRD) data were collected using a Philips X'Pert diffractometer using $\mathrm{Cu} K \alpha$ radiation. The magnetic properties were measured with a physical properties measurement system (PPMS) from Quantum Design. The heterojunction devices were constructed using $\mathrm{Al} / \mathrm{Au}$ contacts, sputtered under a base pressure in the order of $10^{-7}$ Torr at room temperature. The $I-V$ curves were obtained using a Keithley 236 Source Measure Unit and an Oxford DC low noise voltage supply and the magnetic field was applied in the plane of the film.

\section{Structural Properties}

The XRD provides an excellent indication that both the $\mathrm{EuO}$ and $\mathrm{Gd}$ doped $\mathrm{EuO}$ films are high quality europium oxide films. Fig. 1 shows the XRD patterns of both $\mathrm{EuO}_{1-\mathrm{x}}$ and $\mathrm{Eu}_{0.96} \mathrm{Gd}_{0.04} \mathrm{O}$ films. No impurity phase was observed in either the $\mathrm{EuO}$ or the $\mathrm{Eu}_{0.96} \mathrm{Gd}_{0.04} \mathrm{O}$ films, where the substrates were annealed at $750^{\circ} \mathrm{C}$ for a shorter time. For $\mathrm{EuO}_{1-\mathrm{x}}$ and $\mathrm{Eu}_{0.96} \mathrm{Gd}_{0.04} \mathrm{O}_{1-\mathrm{x}}$ films, there is evidence of silicide formation 


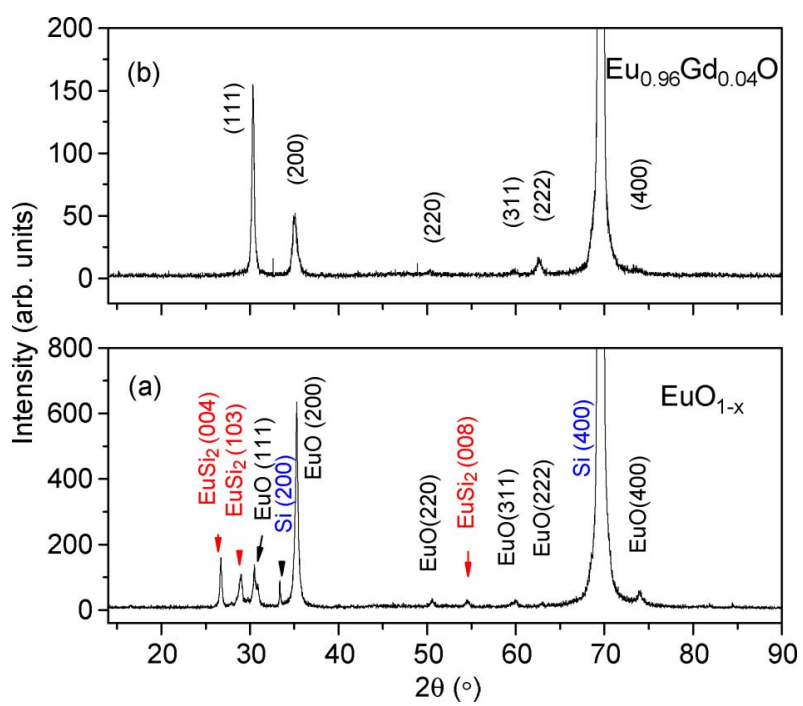

Fig. 1. XRD patterns of $\mathrm{EuO}_{1-\mathrm{x}}$ and $\mathrm{Eu}_{0.96} \mathrm{Gd}_{0.04} \mathrm{O}$ film. A change in the textured growth from the (100) for $\mathrm{EuO}_{1-\mathrm{x}}$ to (111) for $\mathrm{Eu}_{0.96} \mathrm{Gd}_{0.04} \mathrm{O}$ is evident.

as peaks of $\mathrm{EuSi}_{2}$ were observed in some films [see Fig. 1(a)]. The substrates were annealed at $750^{\circ} \mathrm{C}$ for a longer time for these oxygen deficient films. Eu in the laser-induced plume tends to react with $\mathrm{Si}$ to formation $\mathrm{EuSi}_{2}$ in this case because it is less likely to have residual $\mathrm{SiO}_{2}$ on the surface of the substrates that prevents the reaction of $\mathrm{Eu}$ and $\mathrm{Si}$.

As can be seen, for the $\mathrm{EuO}_{1-\mathrm{x}}$ films, the XRD is dominated by the (200) diffraction line as expected from prior work [3], [11]. For the $\mathrm{Eu}_{0.96} \mathrm{Gd}_{0.04} \mathrm{O}$ films the dominant XRD feature is (111) indicating that the preferential texture growth has altered with just this small amount of $\mathrm{Gd}$ doping.

The lattice constants $a$ determined from the (200)/(111) peaks for the Gd-doped, oxygen deficient, and stoichiometric EuO are consistent with the presence of $\mathrm{Gd}$ and $\mathrm{O}$ vacancies. While $a=0.5131 \mathrm{~nm}$ for $\mathrm{EuO}$, this lattice constant decreases to $0.5106 \mathrm{~nm}$ for $\mathrm{EuO}_{1-\mathrm{x}}$. Upon Gd doping, the unreduced sample $\mathrm{Eu}_{0.96} \mathrm{Gd}_{0.04} \mathrm{O}$ has a lattice constant of $a=0.5118 \mathrm{~nm}$ because of the smaller Gd radius, but this value decreases further to $0.5091 \mathrm{~nm}$ for $\mathrm{Eu}_{0.96} \mathrm{Gd}_{0.04} \mathrm{O}_{1-\mathrm{x}}$ when the film is oxygen deficient. Evidently $4 \%$ Gd doping induces a $0.0013-0.0015 \mathrm{~nm}$ reduction in the lattice parameter while oxygen vacancies independently result in a reduction of $0.0025-0.0027 \mathrm{~nm}$. Fig. 2 illustrates the changes in lattice constant $a$ with an increase oxygen vacancies and Gd doping.

While the changes in lattice constant are expected from lattice change, the changes in texture growth are a little unusual, but could be the result of a change in interfacial strain, although small. Although we do not believe these changes are a result of a crystallographic phase change, as with the inclusion of $4 \%$ of $\mathrm{Gd}$ in the cubic $(\mathrm{Fm} 3 \mathrm{~m})$ lattice $\mathrm{EuO}$ is expected to remain a soluble random alloy of the same crystal structure [15], such a possibility cannot be ruled out. Our results suggest PLD is an applicable method for growing doped and undoped EuO films and opens a new simple route to prepare this interesting material. This may be very valuable for spintronic device applications as $\mathrm{EuO}$ and doped films are good candidates for barrier materials in spin filters and for injectors in spin injection devices.

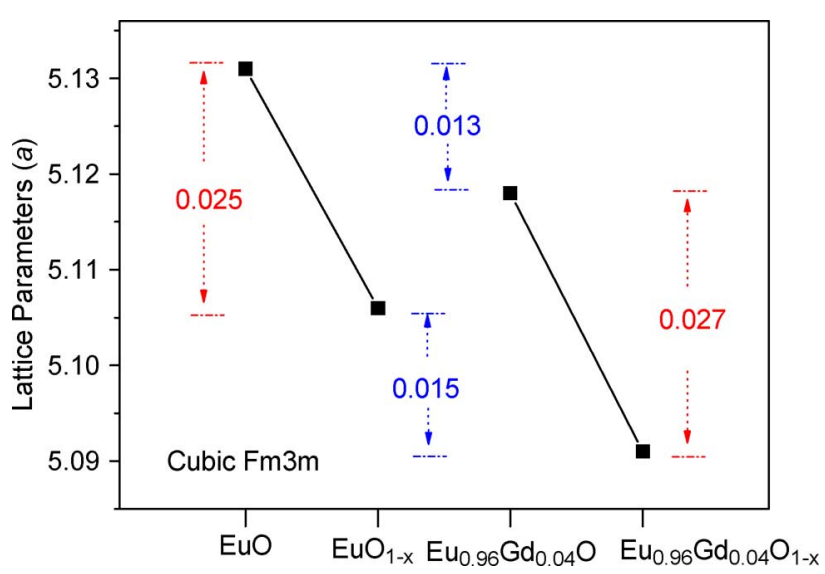

Fig. 2. Lattice parameter $a$ (in $\AA$ ) of the stoichiometric $\mathrm{EuO}$, reduced $\mathrm{EuO}_{1-\mathrm{x}}$, Gd doped unreduced $\mathrm{Eu}_{0.96} \mathrm{Gd}_{0.04} \mathrm{O}$ and $\mathrm{Eu}_{0.96} \mathrm{Gd}_{0.04} \mathrm{O}_{1-\mathrm{x}}$, determined from (200) and (111) diffraction peaks.

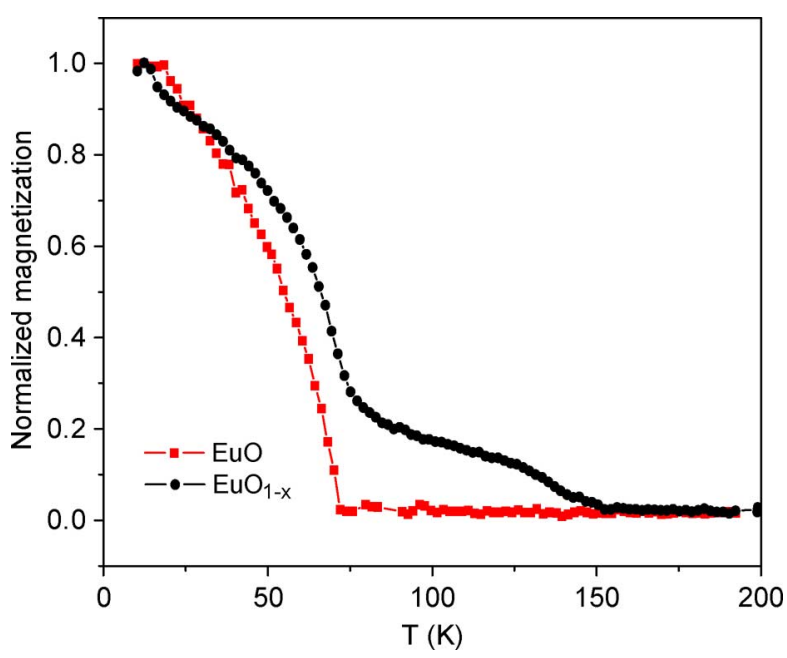

Fig. 3. Magnetization as a function of temperature of EuO films grown by PLD in vacuum and under $\mathrm{H}_{2}$ flow. The latter shows the characteristic double-dome curve of an oxygen deficient $\mathrm{EuO}$ (see text).

\section{MAGNETIC PROPERTIES}

Fig. 3 shows the magnetization versus temperature $M(T)$ curves of $\mathrm{EuO}$ and $\mathrm{EuO}_{1-\mathrm{x}}$ films measured at $100 \mathrm{Oe}$, where the magnetization curves are normalized to unity at $10 \mathrm{~K}$. The ferromagnetic transition of the stoichiometric $\mathrm{EuO}$ is at about $70 \mathrm{~K}$, but the reduced $\mathrm{EuO}_{1-\mathrm{x}}$ has a $\mathrm{T}_{\mathrm{C}}$ as high as $150 \mathrm{~K}$. Fig. 4 shows the magnetic hysteresis curves for the oxygen deficient $\mathrm{EuO}$ at $10 \mathrm{~K}$. Open hysteresis loops were apparent over the whole temperature range though the coercivity of the films decreases with increasing temperature from 70 Oe at $10 \mathrm{~K}$ to $20 \mathrm{Oe}$ at $100 \mathrm{~K}$. For stoichiometric grown $\mathrm{EuO}$, the match to the expected transition temperature (69 K [16]) as well as the XRD results suggest that high quality stoichometric EuO film can be prepared using PLD with metal targets. Our results also demonstrate that we have obtained stoichiometric or oxygen deficient europium oxide films by using PLD through alterations in the conditions for pretreatment of the Si wafer and during the deposition. 


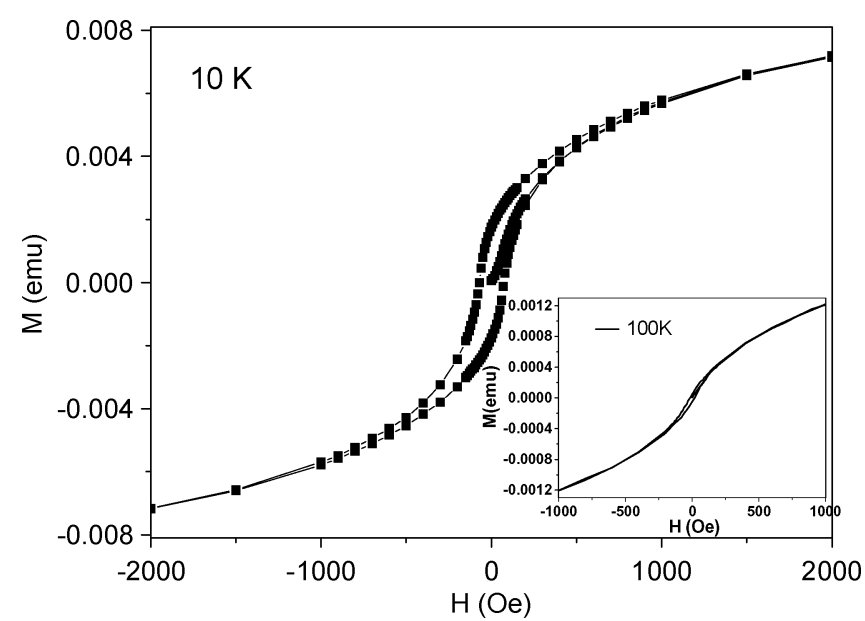

Fig. 4. Magnetic hysteresis curve at $10 \mathrm{~K}$ for the oxygen deficient EuO. Insets show the magnetic hysteresis at $100 \mathrm{~K}$.

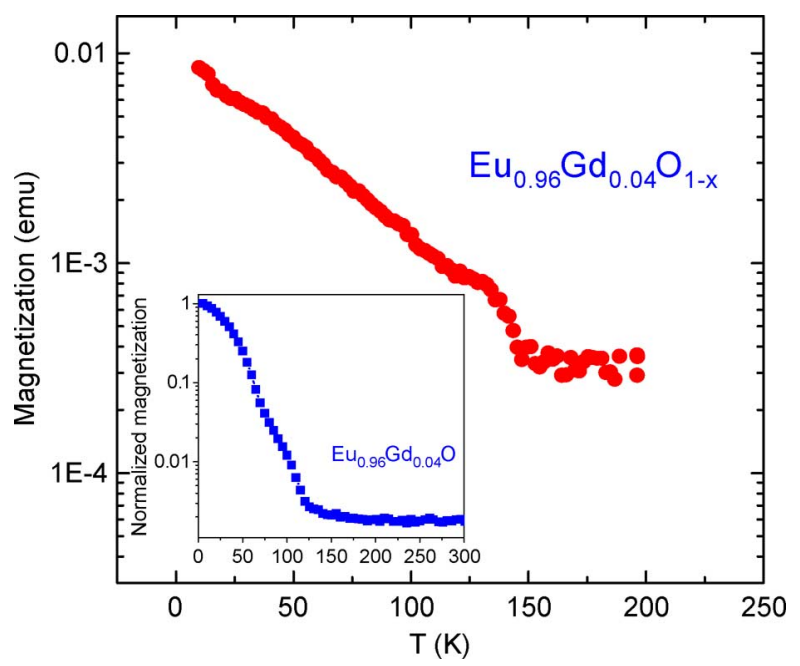

Fig. 5. Magnetization as a function of temperature for $\mathrm{Eu}_{0.96} \mathrm{Gd}_{0.04} \mathrm{O}_{1-\mathrm{x}}$, and inset shows the same magnetization plot for $\mathrm{Eu}_{0.96} \mathrm{Gd}_{0.04} \mathrm{O}$. The magnetization is plotted on a logarithmic scale. The $\mathrm{T}_{\mathrm{C}}$ of Gd-doped un-reduced $\mathrm{Eu}_{0.96} \mathrm{Gd}_{0.04} \mathrm{O}$ is about $120 \mathrm{~K}$, while that of the reduced sample $\mathrm{Eu}_{0.96} \mathrm{Gd}_{0.04} \mathrm{O}_{1-\mathrm{x}}$ is about $145 \mathrm{~K}$.

It is clear from our results (Fig. 3) and those of others [4], [7], [8] that $\mathrm{EuO}_{1-\mathrm{x}}$ exhibits a very much enhanced Curie temperature of about $150 \mathrm{~K}$ with a distinct shape in magnetization versus temperature, i.e., $\mathrm{M}(\mathrm{T})$. A drop in magnetization in the vicinity of $70 \mathrm{~K}$, with an more complete quenching of the magnetization at high temperatures is also observed with temperature dependent magnetization $\mathrm{M}(\mathrm{T})$ of $\mathrm{Eu}_{0.96} \mathrm{Gd}_{0.04} \mathrm{O}$ films, as seen in Fig. 5, inset.

The "double-dome" feature in $\mathrm{M}(\mathrm{T})$ and higher $\mathrm{T}_{\mathrm{C}}$ are characteristic of $\mathrm{EuO}_{1-\mathrm{x}}$ and are not the result of impurities. Other than $\mathrm{Eu}$, no appreciable quantities of other rare earth impurities were present in our $\mathrm{EuO}_{1-\mathrm{x}}$ films. It appears, nonetheless, that either the doping by $\mathrm{O}$ vacancies or the addition of large local moment impurities (Gd) enhances the Curie temperature. It may well be that existence of impurity levels [17], [18] inside the semiconducting gap or an increase in the number of carrier electrons result in the distinct shape of the magnetization $M(T)$.

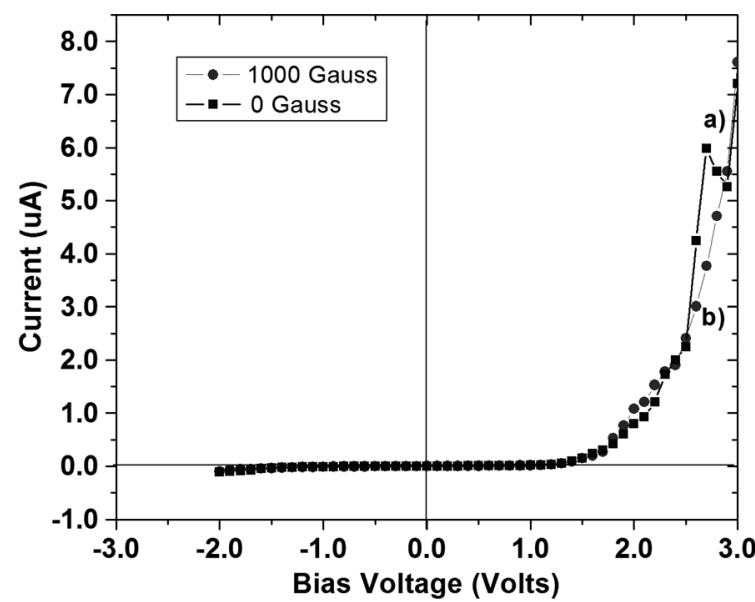

Fig. 6. The magnetization dependent Esaki tunnel diode like behavior for an $\mathrm{Eu}_{0.96} \mathrm{Gd}_{0.04} \mathrm{O}$ on $\mathrm{Si}(100)$ heterostructure. The $I-V$ curve (a) for zero applied magnetic field exhibits negative differential resistance at room temperature at less than $3 \mathrm{~V}$ forward bias. The negative differential resistance is not evident in an applied magnetic field as shown in (b).

The high magnetization below $69-70 \mathrm{~K}$ is likely the result of the large Eu moments, as this is the Curie temperature of stoichiometric EuO. It is expected that the magnetization of $\mathrm{EuO}$ arises from the $4 \mathrm{f}^{7}$ electron configuration of $\mathrm{Eu}^{2+}$. This appears to dominate the magnetization below 69 to $70 \mathrm{~K}$.

At 69 to $70 \mathrm{~K}$, there is a drop in magnetization. Above $70 \mathrm{~K}$, the magnetization may be dominated by the on site polarization of conduction electrons as the carrier densities may be enhanced by oxygen vacancies or Gd doping. While Matsumoto et al. has suggested that the donor levels might be different for Gd doping and oxygen vacancies [4], the overall effect on the temperature dependent magnetization has similarities for both Gd-doped and oxygen deficient films.

Both oxygen deficiencies and the addition of Gd are expected to be n-type donors in EuO, i.e., both add donor states. Both impurities may well act in concert, although differently. While the $\mathrm{T}_{\mathrm{C}}$ of $\mathrm{Gd}$-doped unreduced $\mathrm{Eu}_{0.96} \mathrm{Gd}_{0.04} \mathrm{O}$ is $120 \mathrm{~K}$ (Fig. 5, and inset, magnetization shown on a logarithmic scale), it increases to $145 \mathrm{~K}$ when the Gd-doped sample is oxygen deficient $\left(\mathrm{Eu}_{0.96} \mathrm{Gd}_{0.04} \mathrm{O}_{1-\mathrm{x}}\right)$. There are evident complexities. The combination of Gd doping and oxygen vacancies may degeneratively dope $\mathrm{Eu}_{0.96} \mathrm{Gd}_{0.04} \mathrm{O}$ across the nonmetal to metal transition. Such degenerative doping of the $\mathrm{EuO}$ is suggested by the negative differential resistance in the forward bias of $\mathrm{Eu}_{0.96} \mathrm{Gd}_{0.04} \mathrm{O}$, with both $\mathrm{Gd}$ doping and oxygen vacancies, reminiscent of an Esaki tunnel diode. Fig. 6 shows the magnetization dependent Esaki tunnel diode like behavior (for zero applied field, at room temperature) for an $\mathrm{Eu}_{0.96} \mathrm{Gd}_{0.04} \mathrm{O}$ on $\mathrm{Si}(100)$ heterostructure even with very few oxygen vacancies. The negative differential resistance disappears under the application of a large magnetic field indicating that the electronic structure at the interface due to the local moments may be a key component in the interfacial band alignments either with the contacts or the $\mathrm{Si}(100)$. The magnetic field was applied in the plane of the films and the measurements shown here were done at room temperature. 


\section{CONCLUSION}

We have prepared high quality europium oxide EuO films on Si(100) wafers via PLD. Both stoichiometric and oxygen deficient films of EuO have been fabricated with and without the addition of $\mathrm{Gd}$ as a dopant. The resulting films exhibit changes in lattice constant, texture growth, and $\mathrm{T}_{\mathrm{C}}$. In spite of alteration of the film by a combination of both oxygen vacancies and $\mathrm{Gd}$ doping, the films remain single phase as determined by XRD. The increase in the $\mathrm{T}_{\mathrm{C}}$ may be attributed to the enhanced magnetic coupling by donor states and/or conduction electrons. In spite of the similarities in the enhancement of $\mathrm{T}_{\mathrm{C}}$, we do expect some differences as a result of Gd doping and oxygen vacancies and suggest that the properties of the carriers induced by Gd doping and oxygen vacancies are different. Due to the exchange splitting of either the conductance band edge or the addition of a defect donor state impurity level, $\mathrm{EuO}_{1-\mathrm{x}}$ is believed to have a high spin polarization [3], [10], [12], [13] but the states introduced by oxygen vacancies may not be identical to those introduced by the inclusion of a large local moment impurity like Gd.

\section{ACKNOWLEDGMENT}

This work was supported by NSF (DMR-0852862) and the Defense Threat Reduction Agency under Grant HDTRA1-07-1-008 and the Nebraska Research Initiative, and the National Natural Science Foundation of China under Grant 10804024.

\section{REFERENCES}

[1] T. S. Santos and J. S. Moodera, Phys. Rev. B, vol. 69, p. 241203, 2004.

[2] J. S. Moodera, T. S. Santos, and T. Nagahama, J. Phys.: Condens. Matter, vol. 19, p. 165202, 2007.

[3] A. Schmehl, V. Vaithyanathan, A. Herrnberger, S. Thiel, C. Richter, M. Liberati, T. Heeg, M. Röckerath, L. F. Kourkoutis, S. Mühlbauer, P. Böni, D. A. Muller, Y. Barash, J. Schubert, Y. Idzerda, J. Mannhart, and D. G. Schlom, Nat. Mater., vol. 6, p. 882, 2007.

[4] T. Matsumoto, K. Yamaguchi, M. Yuri, K. Kawaguchi, N. Koshizaki, and K. Yamada, J. Phys.: Condens. Matter, vol. 16, p. 6017, 2004.

[5] M. W. Shafer and T. R. McGuire, J. Appl. Phys., vol. 39, p. 588, 1968.

[6] H. Ott, S. J. Heise, R. Sutarto, Z. Hu, C. F. Chang, H. H. Hsieh, H.-J. Lin, C. T. Chen, and L. H. Tjeng, Phys. Rev. B, vol. 73, p. 094407, 2006.

[7] A. S. Borukhovich, V. G. Bamburov, and A. A. Sidorov, J. Magn. Magn. Mater., vol. 73, p. 106, 1988.

[8] O. Massenet, Y. Capoimont, and N. Van Dang, J. Appl. Phys., vol. 45, p. 3593, 1974.

[9] M. R. Oliver, J. O. Dimmock, A. L. McWhorter, and T. B. Reed, Phys. Rev. B, vol. 5, p. 1078, 1972.

[10] R. P. Panguluri, T. S. Santos, E. Negusse, J. Dvorak, Y. Idzerda, J. S. Moodera, and B. Nadgorny, Phys. Rev. B, vol. 78, p. 125307, 2008.

[11] J. Lettieri, V. Vaithyanathan, S. K. Eah, J. Stephens, V. Sih, D. D. Awschalom, J. Levy, and D. G. Schlom, Appl. Phys. Lett., vol. 83, p. 975, 2003.

[12] K. Sattler and H. C. Siegmann, Phys. Rev. Lett., vol. 29, p. 1565, 1972.

[13] P. G. Steeneken, L. H. Tjeng, I. Elfimov, G. A. Sawatzky, G. Ghiringhelli, N. B. Brookes, and D.-J. Huang, Phys. Rev. Lett., vol. 88, p. 047201, 2002.

[14] H. Ott, S. J. Heise, R. Sutarto, Z. Hu, C. F. Chang, H. H. Hsieh, H.-J. Lin, C. T. Chen, and L. H. Tjeng, Phys. Rev. B, vol. 73, p. 094407 , 2006.

[15] J. M. An, S. Barabash, and K. D. Belashchenko, in preparation.

[16] A. Mauger and C. Godart, Phys. Rep., vol. 141, p. 51, 1986.

[17] M. Arnold and J. Kroha, Phys. Rev. Lett., vol. 100, p. 046404, 2008.

[18] P. Sinjukow and W. Nolting, Phys. Rev. B, vol. 69, p. $214432,2004$. 